\title{
Influence of substratum position and acquired pellicle on Candida albicans biofilm
}

Indira Moraes Gomes Cavalcanti Wander José da Silva

Silvia Carneiro de Lucena

Camila Cordeiro Pousa

Altair Antoninha Del Bel Cury

Department of Prosthodontics and Periodontology, Piracicaba Dental School, Univ of Campinas - UNICAMP, Piracicaba, SP, Brazil.
Declaration of Interests: The authors certify that they have no commercial or associative interest that represents a conflict of interest in connection with the manuscript.

Corresponding Author:

Altair Antoninha Del Bel Cury

E-mail:altcury@fop.unicamp.br

Submitted: Nov 13, 2012

Accepted for publication: Mar 13, 2013

Last revision: Apr 03, 2013
Abstract: The purpose of this study was to evaluate the influence of the substratum position and the saliva acquired pellicle (AP) on Candida albicans biofilm development. Poly (methylmethacrylate) (PMMA) disks were fabricated and randomly allocated to experimental groups: HNP (disks placed in a horizontal position and uncoated by pellicle), VNP (disks placed in a vertical position and uncoated by pellicle), HCP (disks placed in a horizontal position and coated by pellicle), and VCP (disks placed in a vertical position and coated by pellicle). Disks were placed in a 24-well plate and a suspension of $10^{7}$ cells $/ \mathrm{mL}$ of Candida albicans was added to each well for biofilm development. The plates were aerobically incubated at $35^{\circ} \mathrm{C}$. The biofilms were evaluated at 1.5 (adhesion time point), 24, 48, 72, and 96 hours. The number of viable cells was quantified in terms of the colony-forming units per milliliter (CFU/mL). Metabolic activity was measured by the XTT assay. The biofilm structure was analyzed by scanning electron microscopy. The data were analyzed by three-way ANOVA followed by Tukey's test, with significance set at 5\%. The vertical groups showed less biofilm formation and lower metabolic activity than the horizontal groups $(p<0.05)$. Significant differences in cell viability and metabolic activity were observed between the adhesion and other time points $(p<0.05)$, but these variables were not affected by the presence of the pellicle $(p>0.05)$. It can be concluded that the substratum position influenced biofilm development.

Descriptors: Biofilms; Saliva; Candida albicans.

\section{Introduction}

Candida albicans biofilm is an organized community enclosed in an extracellular matrix attached to biotic tissues and abiotic surfaces. ${ }^{1}$ When exposed to the oral environment, prosthetic materials can be a viable substratum for Candida colonization and in an unbalanced oral situation, host factors, favorable substratum and the presence of biofilm contribute to the development of denture-related stomatitis. ${ }^{1,2}$

Candida has been shown to adhere to poly (methylmethacrylate) (PMMA), the material used to fabricate dentures. ${ }^{1,2}$ Several factors, such as the surface roughness $\left(\mathrm{R}_{\mathrm{a}}\right)$, material composition, and exposed area, have been implicated in its colonization..$^{2-4}$ Among the cited factors, the exposed area has not been well explored and it is important to point that the area exposed to biofilm development is connected to the substratum position 
such as either horizontally or vertically. The amount of biofilm formed in a substratum horizontally positioned can be influenced by gravity, which facilitates the deposition of cells onto the surface. In contrast in the vertical position, gravity may impair the cell adhesion. ${ }^{2,4}$ Although the substratum position is known to be related to biofilm formation, how it influences biofilm development has not been thoroughly reported.

Another factor that influences biofilm development is the presence of an acquired pellicle (AP) on the denture material surface. The AP is a conditioning film that forms immediately after the substratum is exposed to the oral environment ${ }^{4,5}$ by the selective adsorption of peptides and proteins from the saliva. PMMA surfaces may be covered by this acellular film, permitting Candida to adhere directly to the PMMA or AP. ${ }^{5,6}$ Numerous in vitro studies have discussed how the substratum and AP influence biofilm formation, ${ }^{4,7}$ but contradictory results have been reported. ${ }^{3,6,8,9}$ Some studies have shown that the presence of an AP reduces the adherence of C. albicans on the acrylic resin surface, ${ }^{3,9}$ whereas other studies did not observe such differences. ${ }^{3,710}$ The presence of a saliva pellicle can alter the substratum properties, such as the surface free energy, because the film composition changes the surface reactivity, which provides different receptor sites for the adherence of microorganisms. ${ }^{5}$ Moreover, $\mathrm{R}_{\mathrm{a}}$ can be modified by an AP once it has masked the substratum roughness, ${ }^{4}$ which may also influence the adhesion of C. albicans.

Given the scarcity of information about the substratum position and the contradictory results about the saliva AP, the purpose of this study was to evaluate the influence of substratum position and the presence of the AP on C. albicans biofilm development.

\section{Methodology Experimental design}

This in vitro study had a randomized and blinded design. Disks of PMMA resin with a standardized $R_{a}$ were fabricated according to the manufacturer's instructions and randomly divided into 4 experimental groups:

- HNP (disks placed in a horizontal position and uncoated by the saliva pellicle),
- VNP (disks placed in a vertical position and uncoated by the saliva pellicle),

- HCP (disks placed in a horizontal position and coated by the saliva pellicle), and

- VCP (disks placed in a vertical position and coated by the saliva pellicle).

The C. albicans reference strain was reactivated and allowed to develop biofilms on disks, which were analyzed at 1.5, 24, 48, 72, and 96 hours. The number of viable cells was expressed in $\mathrm{CFU} / \mathrm{mL}$, the bioactivity was determined by XTT (sodium 3'-[1-(phenylaminocarbonyl)-3,4-tetrazolium]-bis (4-methoxy-6-nitro) benzene sulfonic acid hydrate) assay and the biofilm structure was analyzed by scanning electron microscopy (SEM).

\section{Preparation of PMMA disks}

Disks were fabricated with PMMA resin (QC-20 PMMA; Dentsply, Weybridge, UK) that was polymerized in a hot water bath with a metal matrix $(10 \mathrm{~mm}$ diameter, $2 \mathrm{~mm}$ thickness), according to the manufacturer's instructions. The disks were immersed in distilled water at $37^{\circ} \mathrm{C}$ for 12 hours for residual monomer release. ${ }^{11}$ They were ground with progressively smooth aluminum oxide papers $(320,400$, and 600 grit) in a horizontal polisher (model APL-4; Arotec, São Paulo, Brazil), thoroughly rinsed, and ultrasonically cleaned (Thornton T740; Thornton-Inpec Eletrônica Ltda., Vinhedo, Brazil) for 10 minutes twice to remove any contaminants from the surface. The disks were disinfected with a $0.5 \% \mathrm{NaClO}$ solution for 5 minutes and dried under aseptic conditions. For groups VNP and VCP, the disks were positioned vertically in wells with holders that allowed both surfaces to be colonized by cells. For groups HNP and HCP, the disks were positioned horizontally at the bottom of each well, such that only the surface exposed to the cells was able to be colonized.

\section{$\mathbf{R}_{\mathrm{a}}$ measurements}

The $\mathrm{R}_{\mathrm{a}}$ of the resin disks was measured with a profilometer (Surfcorder SE 1700; Kosaka Laboratory, Kosaka, Japan) with $0.01 \mathrm{~mm}$ resolution, calibrated at a specimen length of $0.8 \mathrm{~mm}, 2.4 \mathrm{~mm}$ percussion of measure at $0.5 \mathrm{~mm} / \mathrm{s}$. For each disk, three 
readings were made, and the mean value was calculated. ${ }^{12}$ The average (mean $\pm S D$ ) $R_{a}$ for all disks was $0.31 \pm 0.02 \mathrm{~mm}$.

\section{Acquired pellicle}

Five healthy volunteers participated in this study, which was approved by the Research and Ethics Committee of FOP/UNICAMP (170/2009). The volunteers provided their written informed consent for participation. Whole stimulated saliva was collected from all volunteers. None of the volunteers were using antibiotics, mouth rinses, or medications that are known to affect the salivary composition or flow.

Saliva was collected during masticatory stimulation with a flexible film (Parafilm M; American Can Co., Neenah, USA). A $50 \mathrm{~mL}$ volume of saliva was clarified in a polypropylene tube by centrifugation at $10,000 \mathrm{~g}$ for 5 minutes at $4^{\circ} \mathrm{C} \cdot .^{13,14}$ For each experiment, the same volume of saliva was collected at the same time of day to standardize the circadian rhythm. The supernatant was filtered through a $0.22 \mu \mathrm{m}$ membrane filter (Corning Inc., Corning, USA) and used immediately. Under aseptic conditions, each disk of the VCP and HCP experimental groups was placed inside a 24 -well plate with $2 \mathrm{~mL}$ of saliva. The plate was incubated for 60 minutes at $35^{\circ} \mathrm{C}$ in an orbital shaker..$^{15}$

\section{Biofilm assay}

Strain reactivation. Biofilm assays were performed with a C. albicans reference strain (ATCC 90028). Prior to each experiment, the strain was grown aerobically on Sabouraud dextrose agar (Difco Laboratories, Detroit, USA) at $35^{\circ} \mathrm{C}$ for 48 hours, inoculated in yeast nitrogen base (YNB) broth medium (Difco Laboratories) that was supplemented with $50 \mathrm{mM}$ glucose, and incubated aerobically at $35^{\circ} \mathrm{C}$ overnight in an orbital shaker (Model NT 151, Kline Shaker; Nova Tecnica Laboratory, Piracicaba, Brazil). The cells were harvested and washed twice with phosphate-buffered solution (PBS, Sigma-Aldrich, St. Louis, USA). ${ }^{16}$

Biofilm development. The cells were resuspended in YNB supplemented with $100 \mathrm{mM}$ glucose. The suspensions were optically adjusted to a density of
$10^{7}$ cells $/ \mathrm{mL}$. A $2 \mathrm{~mL}$ volume of the suspension was added to each well of a 24-well plate containing an experimental disk. The plate was incubated under agitation at $35^{\circ} \mathrm{C}$ for 1.5 hours $^{17}$ (adhesion). Then, the disks were gently washed twice with $2 \mathrm{~mL}$ of PBS and $2 \mathrm{~mL}$ of new YNB medium with $100 \mathrm{mM}$ glucose. The disks were added to a new 24-well plate and incubated for 24, 48, 72, and 96 hours, with a gentle wash every 24 hours before changing the medium.

\section{Biofilm analysis}

Biofilm viability. After each time point, each disk was inserted into a polypropylene tube with $3 \mathrm{ml}$ of PBS and sonicated at $7 \mathrm{~W}$ for 30 seconds for biofilm collection. A $20-\mu \mathrm{L}$ aliquot of the homogenized suspension was serially diluted, plated on Sabouraud dextrose agar, and incubated aerobically for 24 to 30 hours at $35^{\circ} \mathrm{C} .{ }^{18}$ The colony-forming units (CFU) were counted with an optical microscope (Leitz Ortholux; LeitzWetzlar, Germany) at a magnification of $10 \times$ and expressed in $\mathrm{CFU} / \mathrm{mL}$.

Bioactivity analysis. Biofilm bioactivity was analyzed by the XTT reduction assay, as previously described. ${ }^{16,17}$ Briefly, XTT solution was prepared by dissolving XTT salt (Sigma-Aldrich) to a final concentration of $1 \mathrm{mg} / \mathrm{mL}$ in PBS containing $200 \mathrm{mM}$ glucose. The solution was filter-sterilized and stored frozen at $-20^{\circ} \mathrm{C}$ until use. A $0.4 \mathrm{mM}$ solution of menadione (Sigma-Aldrich) in acetone was prepared before each assay. The XTT solution was mixed with the menadione solution at a ratio of 20:1 (v/v). The resin disks with biofilm were placed in a 24 -well plate, and $2 \mathrm{~mL}$ of the XTT solution were added to each well. The plates were covered with aluminum foil and incubated at $35^{\circ} \mathrm{C}$ in the dark under agitation for 3 hours. Afterwards, the solution was centrifuged, and $1.5 \mathrm{~mL}$ were transferred to a cuvette for reading on a spectrophotometer (Beckman Coulter, Indianapolis, USA) recorded at $490 \mathrm{~nm}$.

Structural analysis. The disks were rinsed twice in phosphate buffer and placed in a 24-well plate for subsequent dehydration and fixation. The disks were fixed in Karnovsky (PBS; pH 7.2) solution overnight, dehydrated in a series of ethanol washes $160 \%, 70 \%$, 
Table 1 - Three-way ANOVA for dependent variables.

\begin{tabular}{l|r|r|r|r|r}
\hline \multicolumn{7}{|c|}{} & df & \multicolumn{1}{c}{ SS } & Means square & F-value & \multicolumn{1}{c}{ P } \\
\hline Source & 1 & 63.873 & 63.873 & 584.78 & $<.0001$ \\
\hline Position & 1 & 0.150 & 0.150 & 1.38 & 0.2476 \\
\hline Pellicle & 4 & 4.779 & 1.195 & 10.94 & $<.0001$ \\
\hline Time point & 4 & 5.022 & 1.256 & 11.50 & $<.0001$ \\
\hline Position*time point & 4 & 0.351 & 0.088 & 0.80 & 0.5306 \\
\hline Time point* pellicle & 1 & 0.116 & 0.116 & 1.06 & 0.3091 \\
\hline Position* pellicle & 4 & 0.487 & 0.122 & 1.11 & 0.3632 \\
\hline Position*time Point* pellicle & 19 & 74.779 & 3.936 & 36.03 & \\
\hline Total & & Metabolic activity & & \\
\hline & 1 & 19.795 & 19.795 & 2097.75 & $<.0001$ \\
\hline Position & 1 & 0.000 & 0.000 & 0.01 & 0.9093 \\
\hline Pellicle & 4 & 0.229 & 0.057 & 6.06 & $<.0001$ \\
\hline Time point & 4 & 0.884 & 0.221 & 23.41 & $<.0001$ \\
\hline Position*time point & 4 & 0.060 & 0.015 & 1.59 & 0.1964 \\
\hline Time point* pellicle & 1 & 0.002 & 0.002 & 0.25 & 0.6221 \\
\hline Position* pellicle & 4 & 0.069 & 0.017 & 1.82 & 0.1434 \\
\hline Position*time Point* pellicle & 19 & 21.038 & 1.107 & 117.34 & $<.0001$ \\
\hline Total & & & &
\end{tabular}

$80 \%, 90 \%$ for 5 minutes and $100 \%$ for 10 minutes), dried under aseptic conditions and gold-sputtered for analysis by SEM (Leo 435 VP, Carl Zeiss SMT, Oberkochen, Germany) at $15 \mathrm{kV}$. Images were obtained at $1,000 \times$ magnification.

\section{Statistical analysis}

Statistical analysis was performed with SAS 9.0 software (SAS Institute, Cary, USA). Dependent variables were substratum position, time and presence of AP. Response variables were CFUs and bioactivity. The normality of the error distribution and the degree of nonconstant variance were checked for each response variable. The cell counts were transformed by logarithm $\left[\log _{10}(\chi)\right]$, as suggested by the software. The data were analyzed by three-way analysis of variance (ANOVA), followed by Tukey's HDS test for comparisons. The significance level was set at $5 \%$.

\section{Results}

Three-way ANOVA showed statistically significant differences for position, time, and interactions between position and time. No significant differences were observed for the pellicle or interactions between pellicle and position, pellicle and time, or their combination (Table 1). The amount of biofilm formed was affected by the substratum position for all time points $(p<.0001)$. Vertical disks showed less biofilm formation than horizontal disks $(p<.0001)$. The adhesion time point differed from the other time points for both groups $(p<.0001)$. However, presence of the AP did not affect biofilm formation in the same position $(p=.2476)$ (Figure 1).

The metabolic activity of horizontal groups was higher compared to that of the vertical groups $(p<.001)$. The metabolic activity at the adhesion time point was higher compared to that at all other time points $(p<.001)$ (Figure 2$)$. This variable was not affected by the presence of AP $(p=.9093)$. In accordance with the amount of biofilm formed and the metabolic activity, the SEM images showed more biofilm for the horizontal group. A difference in the amount of biofilm was also observed between the adhesion time point and other time points (Figure 3). 
Figure 1 - CFU $\left(10^{5}\right.$ cells $\left./ \mathrm{mL}\right)$ for each time point in the presence or absence of acquired pellicle in the horizontal and vertical positions (mean \pm SD; $n=9$ ). HCP, HNP:

Disks placed horizontally and coated or uncoated by saliva pellicle. VCP, VNP: Disks placed vertically and coated or uncoated by saliva pellicle. Uppercase letters indicate significant differences between horizontal (A) and vertical (B) groups. *Significant differences between adhesion and other time points. Tukey's test $(p<0.05)$.

Figure 2 - Metabolic activity optical density (O.D; $490 \mathrm{~nm}$; mean $\pm \mathrm{SD} ; n=9$ ). HCP, HNP:

Disks placed horizontally and coated or uncoated by saliva pellicle. VCP, VNP: Disks placed vertically and coated or uncoated by saliva pellicle. Uppercase letters indicate significant differences between horizontal (A) and vertical (B) groups. *Significant differences between adhesion and other time points. Tukey's test $(p<0.05)$.
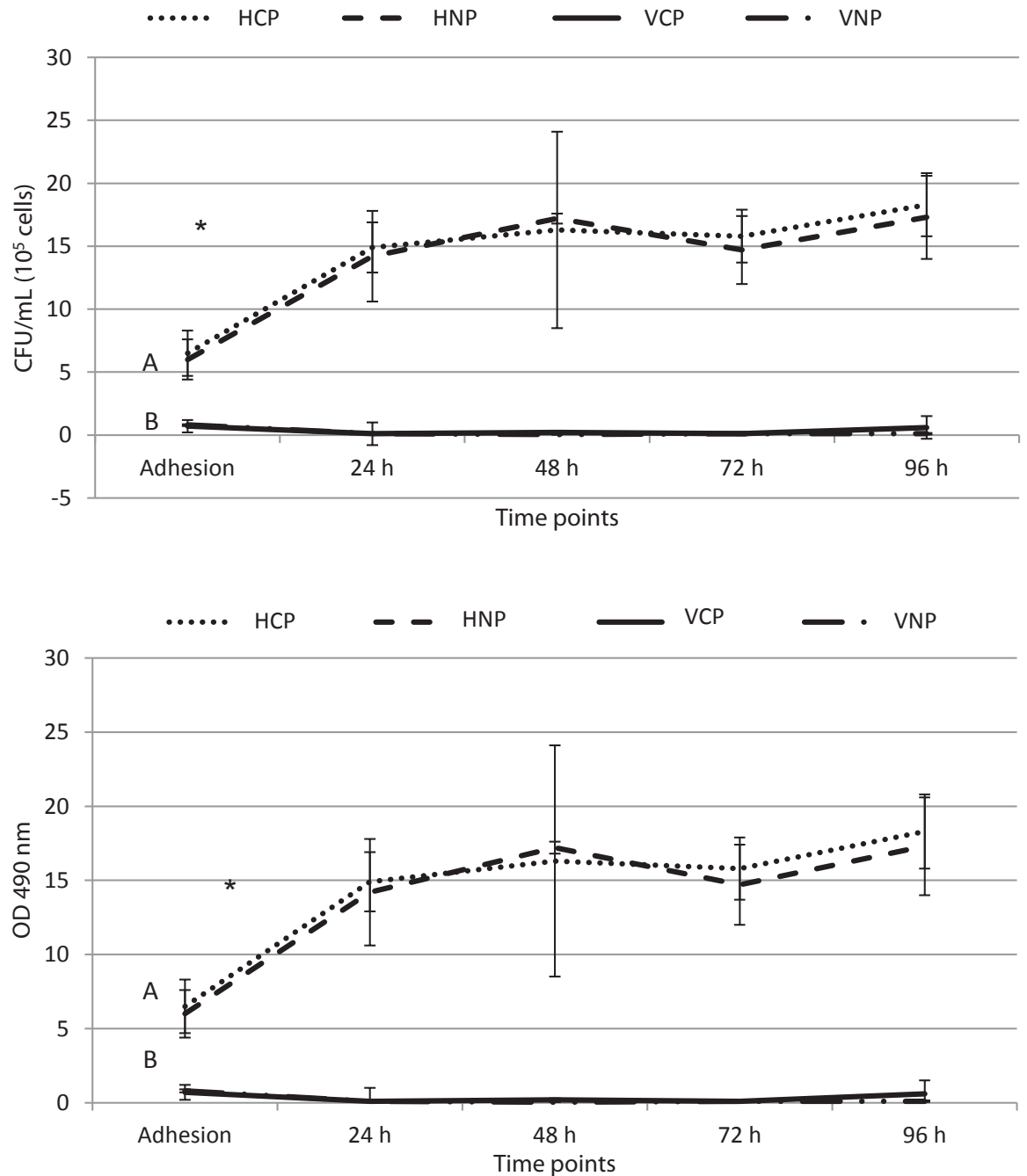

\section{Discussion}

Although the substratum position is known to influence microbial colonization, this variable has not been well characterized. Therefore, the purpose of this in vitro study was to evaluate whether the substratum position and the presence of AP would influence C. albicans biofilm formation. The results showed that the substratum position influenced the amount of biofilm formation. Although the exposed area of the vertically positioned disks was twice the exposed area of the horizontally positioned disks, the number of viable cells on the vertical disks was less than on the horizontals. This result was supported by the reduced metabolic activity of the biofilm formed in the vertical position compared to the horizontal position and by the
SEM images.

These results can be explained by physical factors, such as gravity, which may impair cell deposition in the vertical position but facilitate the deposition horizontally. ${ }^{19}$ Previous studies have simulated microgravity conditions to show that gravity influences biofilm development, suggesting that gravity should be considered as an important factor in biofilm development studies. ${ }^{20,21}$ Future studies are needed to determine whether the adhesive mechanisms of C. albicans are influenced by the substratum position and whether factors other than gravity are involved in the reduced biofilm formation of the vertical position.

The presence of the AP did not influence the amount of biofilm formed in either position or the 

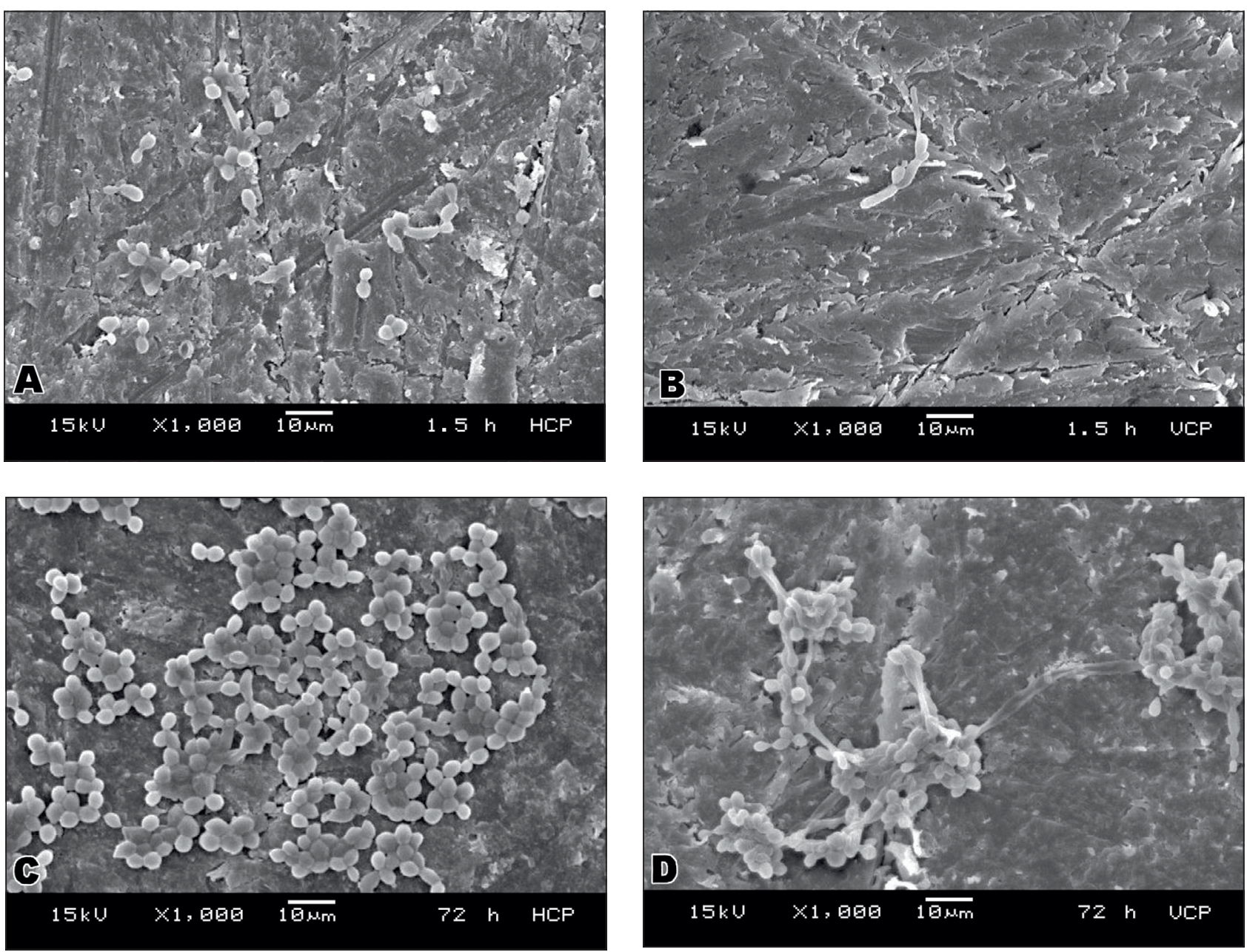

Figure 3 - SEM visualization of biofilm developed on the resin disk surfaces. (A) Disk placed in a horizontal or (B) vertical position, at the adhesion time point (1.5 hours). (C) Disk placed in a horizontal position or (D) vertical position, after 72 hours of biofilm formation.

metabolic activity. It might be hypothesized that the substratum position is a more important factor for biofilm formation than the AP. Previous studies of salivary AP without the influence of the substratum position have noted significant differences, suggesting that saliva is an important modifying factor for surface properties and, consequently, for biofilm development. ${ }^{7,9,15,22}$ However, divergent findings regarding the role of the AP on biofilm formation have been reported, ${ }^{3,5,6,10}$ which may be due to the use of different methodological protocols and saliva collection methods (stimulated or unstimulated), conditions that can change the composition and viscosity of saliva ${ }^{6,10,13}$ and influence biofilm development. ${ }^{6,13}$

A previous study ${ }^{8}$ using stimulated saliva verified that the presence of an AP increased C. albicans adherence to acrylic resin, which is not consistent with our results. In studies using whole and unstimulated saliva, no effect was observed on biofilm formation in the presence of an AP. ${ }^{3,7,10}$ Regardless of whether stimulated or unstimulated saliva is used, the presence of an AP is related to the modification of substratum properties, such as the surface free energy and surface reactivity, which provides different receptor sites for microorganisms. ${ }^{5,22}$ However, in the present study, the vertical positioning may have overcome the effects 
of the AP. Further studies are needed to investigate whether other dental materials with different surfaces properties can produce the same results for $C$. albicans biofilm formation.

\section{References}

1. Sereviratne CJ, Jin L, Samaranayake LP. Biofilm lifestyle of Candida: a mini review. Oral Dis. 2008 Oct;14(7):582-90.

2. Luo G, Samaranayake LP. Candida glabrata, an emerging fungal pathogen, exhibits superior relative cell surface hydrophobicity and adhesion to denture acrylic surfaces compared with Candida albicans. APMIS. 2002 Sep;110(9):601-10.

3. Zaperini CA, Machado AL, Vergani CE, Pavarina AC, Giampaolo ET, Cruz NC. Adherence in vitro of Candida albicans to plasma treated acrylic resin. Effect of plasma parameters, surface roughness and salivary pellicle. Arch Oral Biol. 2010 Oct;55(10):763-70. Epub 2010 Jul 27.

4. Radford DR, Sweet SP, Challacombe SJ, Walter JD. Adherence of Candida albicans to denture-base materials with different surface finishes. J Dent. 1998 Sep;26(7):577-83.

5. Pereira-Cenci T, Del Bel Cury AA, Crielaard W, Ten Cate JM. Development of candida-associated denture stomatitis: new insights. J Appl Oral Sci. 2008 Mar-Apr;16(2):86-94.

6. Pereira-Cenci T, Cury AA, Cenci MS, Rodrigues-Garcia RC. In vitro Candida colonization on acrylic resins and denture liners: influence of surface free energy, roughness, saliva, and adhering bacteria. Int J Prosthodont. 2007 MayJun;20(3):308-10.

7. San Millan R, Elguezabal N, Regulez P, Moragues MD, Quindos G, Ponton J. Effect of salivary secretory IgA on the adhesion of Candida albicans to polystyrene. Microbiology. 2000 Sep;146(Pt 9):2105-12.

8. Vasilas A, Molina L, Hoffman M, Haidaris CG. The influence of morphological variation on Candida albicans adhesion to denture acrylic in vitro. Arch Oral Biol. 1992 Aug;37(8):613-22.

9. Moura JS, Silva WJ, Pereira T, Del Bel Cury AA, Rodrigues Garcia RCM. Influence of acrylic resin polymerization methods and saliva on the adherence of four Candida species. J Prosthet Dent. 2006 Sep;96(3):205-11.

10. Jin Y, Samaranayake LP, Samaranayake Y, Yip HK. Biofilm formation of Candida albicans is variably affected by saliva and dietary sugars. Arch Oral Biol. 2004 Oct;49(10):789-98.

11. Oliveira VM, Leon BL, Del Bel Cury AA, Consani S. Influence of number and position of flasks in the monomer release,

\section{Conclusion}

Considering the limitations of this study, it can be concluded that the substratum position influenced the development of C. albicans biofilm.

Knoop hardness and porosity of a microwave-cured acrylic resin. J Oral Rehabil. 2003 Nov;30(11):1104-8.

12. Verran J, Maryan CJ. Retention of Candida albicans on acrylic resin and silicone of different surface topography. J Prosthet Dent. 1997 May;77(5):535-9.

13. Aps JK, Martens LC. Review: the physiology of saliva and transfer of drugs into saliva. Forensic Sci Int. 2005 Jun 10;150(2-3):119-31.

14. Thein ZM, Samaranayake YH, Samaranayake LP. Characteristics of dual species Candida biofilms on denture acrylic surfaces. Arch Oral Biol. 2007 Dec;52(12):1200-8. Epub 2007 Aug 6.

15. Nikawa H, Nishimura H, Makihira S, Hamada T, Sadamori $S$, Samaranayake LP. Effects of serum concentration on Candida biofilm formation on acrylic surfaces. Mycoses. 2000 May;43(3-4):139-43.

16. da Silva WJ, Seneviratne J, Parahitiyawa N, Rosa EA, Samaranayake LP, Del Bel Cury AA. Improvement of XTT assay performance for studies involving Candida albicans biofilms. Braz Dent J. 2008;19(4):364-9.

17. Gomes PN, Silva WJ, Pousa CC, Del Bel Cury AA. Bioactivity and cellular structure of Candida albicans and Candida glabrata biofilms grown in the presence of fluconazole. Arch Oral Biol. 2011 Nov;56(11):1274-81. Epub 2011 May 6.

18. Ramage G, Tomsett K, Wickes BL, Lopez-Ribot JL, Redding SW. Denture stomatitis: a role for Candida biofilms. Oral Surg Oral Med Oral Pathol Oral Radiol Endod. 2004 Jul;98(1):53-9.

19. Soll DR. Candida commensalism and virulence: the evolution of phenotypic plasticity. Acta Trop. 2002 Feb;81(2):101-10.

20. McLean RJ, Cassanto JM, Barnes MB, Koo JH. Bacterial biofilm formation under microgravity conditions. FEMS Microbiol Lett. 2001 Feb 20;195(2):115-9.

21. Lynch SV, Mukundakrishnan K, Benoit MR, Ayyaswamy PS, Matin A. Escherichia coli biofilms formed under low-shear modeled microgravity in a ground-based system. Appl Environ Microbiol. 2006 Dec;72(12):7701-10. Epub 2006 Oct 6.

22. Sipahi C, Anil N, Bayramli E. The effect of acquired salivary pellicle on the surface free energy and wettability of different denture base materials. J Dent. 2001 Mar;29(3):197-204. 